TRANSACTIONS OF THE

AMERICAN MATHEMATICAL SOCIETY

Volume 352, Number 7 , Pages 3239-3263

S 0002-9947(00)02671-4

Article electronically published on March 21, 2000

\title{
STEINER TYPE FORMULAE AND WEIGHTED MEASURES OF SINGULARITIES FOR SEMI-CONVEX FUNCTIONS
}

\author{
ANDREA COLESANTI AND DANIEL HUG
}

\begin{abstract}
For a given convex (semi-convex) function $u$, defined on a nonempty open convex set $\Omega \subset \mathbf{R}^{n}$, we establish a local Steiner type formula, the coefficients of which are nonnegative (signed) Borel measures. We also determine explicit integral representations for these coefficient measures, which are similar to the integral representations for the curvature measures of convex bodies (and, more generally, of sets with positive reach). We prove that, for $r \in\{0, \ldots, n\}$, the $r$-th coefficient measure of the local Steiner formula for $u$, restricted to the set of $r$-singular points of $u$, is absolutely continuous with respect to the $r$-dimensional Hausdorff measure, and that its density is the $(n-r)$-dimensional Hausdorff measure of the subgradient of $u$.

As an application, under the assumptions that $u$ is convex and Lipschitz, and $\Omega$ is bounded, we get sharp estimates for certain weighted Hausdorff measures of the sets of $r$-singular points of $u$. Such estimates depend on the Lipschitz constant of $u$ and on the quermassintegrals of the topological closure of $\Omega$.
\end{abstract}

\section{INTRODUCTION}

The structure of the set of singular points of a convex function $u$, defined on an open convex set $\Omega \subset \mathbb{R}^{n}$, presents interesting aspects, both analytic and geometric. A first step in the study of this set is to consider the following sets $\Sigma^{r}(u), r \in$ $\{0, \ldots, n\}$, of $r$-singular points of $u$ :

$$
\Sigma^{r}(u):=\{x \in \Omega: \operatorname{dim} \partial u(x) \geq n-r\},
$$

where $\partial u(x)$ denotes the subgradient of $u$ at $x$. Alberti, Ambrosio \& Cannarsa [1] proved that, for every $r \in\{0, \ldots, n\}, \Sigma^{r}(u)$ can be covered by countably many $r$-dimensional submanifolds of class $C^{1}$, at least up to a set of zero $r$-dimensional Hausdorff measure. In particular, it follows that the Hausdorff dimension of $\Sigma^{r}(u)$ is at most $r$.

Quite simple examples show that the $r$-dimensional Hausdorff measure of $\Sigma^{r}(u)$ can be infinite, for some convex function $u: \Omega \rightarrow \mathbb{R}$, even if $\Omega$ is bounded. Therefore, a weighted $r$-dimensional Hausdorff measure of $\Sigma^{r}(u)$ will be considered, where the weight to be assigned to a point $x \in \Sigma^{r}(u)$ is the $(n-r)$-dimensional Hausdorff

Received by the editors December 30, 1996.

2000 Mathematics Subject Classification. Primary 26B25, 52A41; Secondary 28A78, 52A20, 49J52, 49Q15.

Key words and phrases. Steiner formula, convex function, semi-convex function, singularities, weighted Hausdorff measures, subgradient map, unit normal bundle, non-smooth analysis.

(C)2000 American Mathematical Society 
measure of $\partial u(x)$. In other words, we investigate the integral

$$
\int_{\Sigma^{r}(u)} \mathcal{H}^{n-r}(\partial u(x)) \mathrm{d} \mathcal{H}^{r}(x),
$$

where $\mathcal{H}^{s}$, for $s \geq 0$, denotes the $s$-dimensional Hausdorff measure. In [1], for a convex and Lipschitz function $u: \Omega \rightarrow \mathbb{R}$, defined on a nonempty, open, bounded and convex set $\Omega \subset \mathbb{R}^{n}$, with Lipschitz constant $L$, and for every $r \in\{0, \ldots, n\}$, the following inequality is proved:

$$
\int_{\Sigma^{r}(u)} \mathcal{H}^{n-r}(\partial u(x)) \mathrm{d} \mathcal{H}^{r}(x) \leq C(L+\operatorname{diam}(\Omega))^{n},
$$

where $C$ is a constant depending on $n$.

In the present paper we establish a sharp upper bound for the integral (1), thus improving (2). Namely, in Theorem 6.3 we prove that

$$
\int_{\Sigma^{r}(u)} \mathcal{H}^{n-r}(\partial u(x)) \mathrm{d} \mathcal{H}^{r}(x) \leq\left(\begin{array}{c}
n \\
r
\end{array}\right) L^{n-r} W_{n-r}(\bar{\Omega}),
$$

for $r \in\{0, \ldots, n\}$. The quantities $W_{i}(\bar{\Omega}), i \in\{0, \ldots, n\}$, denote the quermassintegrals of the topological closure $\bar{\Omega}$ of $\Omega$. In Section 7 , for every open bounded convex set $\Omega$, and for every $L \geq 0$ and $\epsilon>0$, we find a convex Lipschitz function $u$ defined on $\Omega$, with Lipschitz constant $L$, such that, for all $r \in\{0, \ldots, n\}$,

$$
\int_{\Sigma^{r}(u)} \mathcal{H}^{n-r}(\partial u(x)) \mathrm{d} \mathcal{H}^{r}(x) \geq\left(\begin{array}{c}
n \\
r
\end{array}\right) L^{n-r} W_{n-r}(\bar{\Omega})-\epsilon .
$$

These results represent a counterpart, in the context of convex Lipschitz functions, of some properties of the sets of singular boundary points of convex bodies, proved in [7] and, in the more general setting of sets with positive reach, in [11]. There, for a convex body $K \subset \mathbb{R}^{n}$, the set $\Sigma^{r}(K)$ of $r$-singular boundary points of $K$ is considered. Recall from 11 that $\Sigma^{r}(K)$ is defined by

$$
\Sigma^{r}(K)=\{x \in \partial K: \operatorname{dim} N(K, x) \geq n-r\},
$$

for $r \in\{0, \ldots, n-1\}$, if $N(K, x)$ denotes the normal cone of $K$ at $x$.

The following inequalities are proved in [7] for $n=3$ and $r=1$, and in [1] for arbitrary $n \geq 2$ and $r \in\{0, \ldots, n-1\}$ :

$$
\int_{\Sigma^{r}(K)} \mathcal{H}^{n-1-r}\left(N(K, x) \cap S^{n-1}\right) \mathrm{d} \mathcal{H}^{r}(x) \leq n\left(\begin{array}{c}
n-1 \\
r
\end{array}\right) W_{n-r}(K),
$$

where $W_{i}(K)$, for $i \in\{0, \ldots, n\}$, are the quermassintegrals of the convex body $K$. Note that the inequalities (4) are sharp; indeed equality holds when $K$ is a polytope.

The proof of the inequalities (3), given in Section 6, requires several preliminary results, which are contained in Sections 3 and 4. The basic ingredient is the local Steiner formula for an arbitrary convex function $u$, which we establish in Section 3. This result is a generalization of Theorem 1.1 in [6]. Whereas approximation of $u$ by special smooth convex functions and weak continuity of coefficient measures were used to prove the Steiner formula in [6], the present approach exploits the connection between analytic properties of the function $u$ and geometric properties 
of its epigraph. This, in addition, leads to explicit integral representations for the coefficient measures, which are measures on the $\sigma$-algebra of Borel sets of $\Omega \times \mathbb{R}^{n}$.

In Section 4, we relate the $r$-th coefficient measure $\Theta_{r}(u, \cdot)$ of the Steiner formula for the convex function $u$ to the integral (1), by proving the formula

$$
\int_{\Sigma^{r}(u)} \mathcal{H}^{n-r}(\partial u(x)) \mathrm{d} \mathcal{H}^{r}(x)=\left(\begin{array}{l}
n \\
r
\end{array}\right) \Theta_{r}\left(u, \Sigma^{r}(u) \times \mathbb{R}^{n}\right),
$$

for $r \in\{0, \ldots, n\}$. More generally, formula (5) can be stated for arbitrary Borel subsets of $\Sigma^{r}(u) \times \mathbb{R}^{n}$. Let us denote by $\pi_{1}: \mathbb{R}^{n} \times \mathbb{R}^{n} \rightarrow \mathbb{R}^{n}$ the projection map $\pi_{1}(x, y):=x$. Then it follows from the extended version of (15) that the restriction of the image measure $F_{n-r}(u, \cdot):=\Theta_{r}(u, \cdot) \circ \pi_{1}^{-1}$ to Borel subsets of $\Sigma^{r}(u)$ is absolutely continuous with respect to the $r$-dimensional Hausdorff measure. Note that a similar result holds in the context of convex bodies. In fact, the restriction of the $r$-th curvature measure of a convex body $K$ to the set $\Sigma^{r}(K)$ is absolutely continuous with respect to the $r$-dimensional Hausdorff measure, and the density can be explicitly determined as well, see [11, Theorem 3.2].

The results of Sections 3 and 4 are extended in Section 5 to semi-convex functions, with no essential difficulty. It is worth remarking that this extension parallels the extension, to sets of positive reach, of several results regarding sets of singular boundary points of convex bodies, see [1]. The close connection between semiconvex functions and sets with positive reach has previously been observed and developed by Bangert [3] and $\mathrm{Fu}[10$.

\section{NotATION AND PRELIMINARIES}

Results for sets with positive reach, and hence especially for convex sets, will play an important rôle in the sequel. The basic theory in this field has been developed by Federer in his classical paper [8]. Later M. Zähle [16] extended and simplified Federer's theory substantially. In this section, we collect some of those facts which will be needed in the following. Details and proofs as well as further references can be found in Zähle [16], see also Kohlmann [12].

The basic setting is given by the Euclidean space $\mathbb{R}^{d}(d \geq 2)$ with scalar product $\langle\cdot, \cdot\rangle$ and norm $|\cdot|$. Henceforth, let $X \subset \mathbb{R}^{d}$ be a nonempty closed set with $X \neq \mathbb{R}^{d}$. For $a \in \mathbb{R}^{d}$ we define $\operatorname{dist}(a, X):=\min \{|a-x|: x \in X\}$. Let $\operatorname{Unp}(X)$ be the set of all $a \in \mathbb{R}^{d}$ for which card $\{x \in X:|a-x|=\operatorname{dist}(a, X)\}=1$, that is, $a \in \operatorname{Unp}(X)$ holds if and only if there exists a unique nearest point $p_{X}(a)$ to $a$ in $X$.

For $x \in X$ we define the number

$$
\operatorname{reach}(X, x):=\sup \{r \geq 0: U(x, r) \subset \operatorname{Unp}(X)\},
$$

where $U(x, r):=\left\{z \in \mathbb{R}^{d}:|z-x|<r\right\}$. Finally, we say that $X$ has positive reach, if

$$
\operatorname{reach}(X):=\inf \{\operatorname{reach}(X, x): x \in X\}>0 .
$$

Examples of sets with $\operatorname{reach}(X)=\infty$ are nonempty closed convex sets $X \subset \mathbb{R}^{d}$ with $X \neq \mathbb{R}^{d}$.

Provided that $\epsilon \in(0, \operatorname{reach}(X))$, the projection map $p_{X}$ is at least defined on $X^{\epsilon}:=\left\{a \in \mathbb{R}^{d}: \operatorname{dist}(a, X) \leq \epsilon\right\}$. In addition, we introduce the maps

$$
\sigma_{X}: X^{\epsilon} \backslash X \rightarrow S^{d-1}, \quad a \mapsto\left|a-p_{X}(a)\right|^{-1}\left(a-p_{X}(a)\right),
$$


and

$$
F_{X}: X^{\epsilon} \backslash X \rightarrow \mathbb{R}^{d} \times S^{d-1}, \quad a \mapsto\left(p_{X}(a), \sigma_{X}(a)\right) .
$$

Denote by $\partial M$ the topological boundary and by $\bar{M}$ the closure of a set $M$. Since $F_{X} \mid \partial X^{\epsilon}$ is a bi-Lipschitz homeomorphism and $\partial X^{\epsilon}$ is a $(d-1)$-dimensional $C^{1}$ submanifold of $\mathbb{R}^{d}$, the image set $\operatorname{Nor}(X):=F_{X}\left(\partial X^{\epsilon}\right)$ is countably $(d-1)$-rectifiable in the sense of Federer. To Federer $9, \S 3.2 .14]$ we refer for all notions of geometric measure theory such as rectifiability, approximate differentials and Jacobians, or approximate tangent vectors.

Assume now that $\epsilon \in(0$, reach $(X))$, and let $y \in \partial X^{\epsilon}$ be such that $\sigma_{X}: \partial X^{\epsilon} \rightarrow$ $S^{d-1}$ is differentiable at $y$. The differential $D \sigma_{X}(y): \sigma_{X}(y)^{\perp} \rightarrow \sigma_{X}(y)^{\perp}$ is a symmetric linear map with eigenvalues $k_{1}(y), \ldots, k_{d-1}(y)$ and corresponding eigenvectors $u_{1}(y), \ldots, u_{d-1}(y)$. Let $(x, v):=F_{X}(y) \in \operatorname{Nor}(X)$. Then one defines

$$
k_{i}(x, v):=\left\{\begin{array}{l}
\frac{k_{i}(y)}{1-\epsilon k_{i}(y)}, \quad \text { if } k_{i}(y)<\epsilon^{-1} \\
\infty, \quad \text { if } k_{i}(y)=\epsilon^{-1}
\end{array}\right.
$$

for $i \in\{1, \ldots, d-1\}$. One can show that this definition is independent of the particular choice of $\epsilon \in(0, \operatorname{reach}(X))$. Moreover, the unit vectors $u_{1}(x+\epsilon v)$, $\ldots, u_{d-1}(x+\epsilon v)$ do not depend on $\epsilon$ and represent an orthonormal basis of $v^{\perp}$. Thus, (generalized) curvatures $k_{i}(x, v), i \in\{1, \ldots, d-1\}$, are defined for all $(x, v) \in \operatorname{Nor}(X)$ such that $\sigma_{X} \mid \partial X^{\delta}$ is differentiable at $(x+\delta v)$ for some (and hence for all $) \delta \in(0, \operatorname{reach}(X))$. In this situation, it is also known that the set of $\left(\mathcal{H}^{d-1}\llcorner\operatorname{Nor}(X), d-1)\right.$ approximate tangent vectors at $(x, v)$ is a $(d-1)$-dimensional linear subspace of $\mathbb{R}^{d} \times \mathbb{R}^{d}$, which is denoted by $\operatorname{Tan}^{d-1}\left(\mathcal{H}^{d-1}\llcorner\operatorname{Nor}(X),(x, v))\right.$. This approximate tangent space is spanned by the orthonormal basis $w_{i}(x, v)$, $i \in\{1, \ldots, d-1\}$, where

$$
w_{i}(x, v):=\left(\frac{1}{\sqrt{1+k_{i}(x, v)^{2}}} u_{i}(x, v), \frac{k_{i}(x, v)}{\sqrt{1+k_{i}(x, v)^{2}}} u_{i}(x, v)\right),
$$

and $u_{i}(x, v):=u_{i}(x+\epsilon v)$ for $i \in\{1, \ldots, d-1\}$. Since $\sigma_{X} \mid \partial X^{\epsilon}$ is a Lipschitz map, the preceding statements hold true for $\mathcal{H}^{d-1}$ almost all $(x, v) \in \operatorname{Nor}(X)$.

Part of the present research is devoted to the investigation of singularities of convex functions, see Section 4. If $u: \Omega \rightarrow \mathbb{R}$ is a convex function defined on a (nonempty) open convex set in $\mathbb{R}^{d}$, then the subgradient $\partial u(x)$ of $u$ at $x \in \Omega$ is defined by

$$
\partial u(x):=\left\{p \in \mathbb{R}^{d}: \forall y \in \Omega u(y)-u(x) \geq\langle p, y-x\rangle\right\} .
$$

It is well known that this is a nonempty compact convex set. A point $x \in \Omega$ is called singular, if $\operatorname{dim} \partial u(x) \geq 1$. In Section 4 we will classify the points $x \in \Omega$ according to the dimension of $\partial u(x)$.

For a set $X$ of positive reach we now introduce the classical notion of a normal cone. This is the notion which should be seen in analogy to the subgradient of a function. The normal cone $N(X, x)$ of $X$ at a boundary point $x \in \partial X$ can be defined by

$$
N(X, x):=\left\{\lambda v \in \mathbb{R}^{d}:(x, v) \in \operatorname{Nor}(X), \lambda \geq 0\right\}
$$


It is easy to see that this is a nonempty closed convex set. Moreover, this definition gives rise to a classification of boundary points according to

$$
\Sigma^{r}(X):=\{x \in \partial X: \operatorname{dim} N(X, x) \geq d-r\},
$$

for $r \in\{0, \ldots, d-1\}$. The set $\Sigma^{r}(X)$ is called the set of $r$-singular boundary points of $X$. It is known that $\Sigma^{r}(X)$ is an $r$-rectifiable Borel set. Recently, geometric and measure theoretic properties of these sets have been investigated in [11].

As far as measure theory is concerned, we write $\mathcal{H}^{r}$ for the $r$-dimensional Hausdorff measure, $r \geq 0$, and $\mathfrak{B}(T)$ denotes the $\sigma$-algebra of Borel sets of a topological space $T$. Our basic reference for results from measure theory is Federer [9] for results about convex sets or functions we usually refer to Schneider [14].

\section{Steiner formulae And COefFicient measures}

In this section, we will establish a general Steiner type formula for an arbitrary convex function $u$. The present result represents a generalization of Theorem 1.1 from [6].

First, let us introduce some more notation and add some preparatory remarks. In the present section, it is always assumed that $\Omega \subset \mathbb{R}^{n}$ is a nonempty open convex set and that $u: \Omega \rightarrow \mathbb{R}$ is a convex function. As usual, the epigraph of $u$ is defined by setting

$$
\operatorname{epi}(u):=\{(x, t) \in \Omega \times \mathbb{R}: t \geq u(x)\} .
$$

The closure of the epigraph of $u, \mathfrak{X}_{u}:=\overline{\operatorname{epi}(u)} \subset \mathbb{R}^{n+1}$, is a nonempty closed convex subset of $\mathbb{R}^{n+1}$, and $\mathfrak{X}_{u} \neq \mathbb{R}^{n+1}$. In particular, we have reach $\left(\mathfrak{X}_{u}\right)=\infty$. Thus, the present setting admits the application of the methods developed by M. Zähle in [16, see also [11].

In order to introduce local parallel sets for the convex function $u$, we consider the (generalized) graph $\Gamma(u)$ of the multivalued subgradient map $\partial u$ of $u$ over $\Omega$, which is given by

$$
\Gamma(u):=\left\{(x, p) \in \Omega \times \mathbb{R}^{n}: p \in \partial u(x)\right\} .
$$

Then, for any $\eta \in \mathfrak{B}\left(\Omega \times \mathbb{R}^{n}\right)$ and $\rho \geq 0$, the local parallel set $P_{\rho}(u, \eta)$ of the convex function $u$,

$$
P_{\rho}(u, \eta):=\left\{x+\rho p \in \mathbb{R}^{n}:(x, p) \in \eta \cap \Gamma(u)\right\},
$$

can be defined. This definition is analogous to the one used in the context of convex bodies, see Schneider [14 §4.1]. We should, however, remark that $P_{\rho}(u, \Omega)$ need neither be convex nor star-shaped in general.

It will be necessary, for the present approach, to obtain an alternative description of these local parallel sets. For that purpose, rather than the unit normal bundle $\operatorname{Nor}\left(\mathfrak{X}_{u}\right)$ of $\mathfrak{X}_{u}$, we shall need the following subset

$$
\operatorname{Nor}(u):=\operatorname{Nor}\left(\mathfrak{X}_{u}\right) \cap\left(\left\{(x, u(x)) \in \mathbb{R}^{n+1}: x \in \Omega\right\} \times S^{n}\right)
$$

of $\mathbb{R}^{n+1} \times \mathbb{R}^{n+1}$, which corresponds to the domain of $u$. The set Nor $(u)$ will be called the unit normal bundle of $u$. Obviously, this is a countably $n$-rectifiable Borel set. A precise correspondence between the sets $\Gamma(u)$ and $\operatorname{Nor}(u)$ is provided by the map

$$
T:\left\{\begin{aligned}
\Gamma(u) \rightarrow \operatorname{Nor}(u), \\
(x, p) \mapsto\left((x, u(x)), \frac{1}{\sqrt{1+|p|^{2}}}(p,-1)\right) .
\end{aligned}\right.
$$


According to Theorem 1.5.11 from Schneider 14 and since $u$ is real-valued and $\Omega$ is open, this map is well-defined and onto. Moreover, it is easy to check that it is also one-to-one.

Let us denote by $\left(E_{1}, \ldots, E_{n+1}\right)$ the standard basis of $\mathbb{R}^{n+1}$. Points and vectors of $\mathbb{R}^{n+1}$ will be denoted by capital letters. Furthermore, we identify $\mathbb{R}^{n}$ and $\mathbb{R}^{n} \times$ $\{0\}$. Then, the inverse map $\tilde{T}$ of $T$ is given by

$$
\tilde{T}:\left\{\begin{array}{l}
\operatorname{Nor}(u) \rightarrow \Gamma(u), \\
(X, V) \mapsto\left(X-\left\langle X, E_{n+1}\right\rangle E_{n+1}, E_{n+1}-\left\langle V, E_{n+1}\right\rangle^{-1} V\right) .
\end{array}\right.
$$

Note that $\left\langle V, E_{n+1}\right\rangle<0$ holds true, if $(X, V) \in \operatorname{Nor}(u)$ for some $X \in \mathbb{R}^{n+1}$. Hence, the maps $T$ and $\tilde{T}$ are mutually inverse homeomorphisms, and locally they are Lipschitz maps. This implies, in particular, that $\Gamma(u)$ is a countably $n$-rectifiable Borel set.

Now, for any $\eta \in \mathfrak{B}\left(\Omega \times \mathbb{R}^{n}\right)$ and $\rho \geq 0$, the local parallel set $P_{\rho}(u, \eta)$ of the convex function $u$ can be parametrized by using the Borel set

$$
\hat{\eta}:=T(\eta \cap \Gamma(u)) \subset \mathbb{R}^{n+1} \times \mathbb{R}^{n+1}
$$

and the transformation $F_{\rho}: \operatorname{Nor}(u) \rightarrow \mathbb{R}^{n}$ which is given by

$$
F_{\rho}:=\pi_{1} \circ \tilde{T}+\rho \pi_{2} \circ \tilde{T},
$$

where the projection maps $\pi_{1}, \pi_{2}: \mathbb{R}^{n} \times \mathbb{R}^{n} \rightarrow \mathbb{R}^{n}$ are defined by setting

$$
\pi_{1}(x, u):=x \quad \text { and } \quad \pi_{2}(x, u):=u .
$$

Note that $F_{\rho}$ is injective for $\rho>0$. This can easily be inferred from Proposition 2.2 of [6]. In fact, this proposition remains true, with the same proof, under the present weaker assumptions, where it is neither assumed that $\Omega$ is bounded nor that $u$ is a Lipschitz map. In fact, these remarks eventually lead to the relation

$$
P_{\rho}(u, \eta)=F_{\rho}(\hat{\eta}) \subset \mathbb{R}^{n},
$$

for any $\eta \in \mathfrak{B}\left(\Omega \times \mathbb{R}^{n}\right)$ and $\rho \geq 0$. Finally, observe that $P_{\rho}(u, \eta)$ is a countably $n$ rectifiable Borel set. A short proof for the fact that $P_{\rho}(u, \eta) \in \mathfrak{B}\left(\mathbb{R}^{n}\right)$ can be given by applying Theorem 8.3.7 of Cohn [5]. For a more elementary approach, one first shows that $P_{\rho}(u, \Omega)$ is a countable union of closed sets, and then uses Proposition 2.2 of $[6]$.

The preceding considerations and Federer's coarea formula [9, Theorem 3.2.22] hence imply that

$$
\begin{aligned}
\mathcal{H}^{n}\left(P_{\rho}(u, \eta)\right) & =\mathcal{H}^{n}\left(F_{\rho}(\operatorname{Nor}(u) \cap \hat{\eta})\right) \\
& =\int_{\operatorname{Nor}(u)} \mathbf{1}_{\hat{\eta}}(X, V) \text { ap } J_{n} F_{\rho}(X, V) \mathrm{d} \mathcal{H}^{n}(X, V) .
\end{aligned}
$$

Here again the injectivity of $F_{\rho}$ is used if $\rho>0$. But (6) is also true for $\rho=0$, since $\partial u(x)$ is single-valued for $\mathcal{H}^{n}$ almost all $x \in \Omega$.

Therefore, in order to obtain the desired Steiner type formula and the integral representation for the coefficient measures, it remains to determine the approximate Jacobian ap $J_{n} F_{\rho}(X, V)$ of the mapping $F_{\rho}$. Before we can express the precise result in Theorem 3.1 below, some of the facts mentioned in Section 2 have to be 
interpreted in the present framework. In particular, note that for $\mathcal{H}^{n}$ almost all $(X, V) \in \operatorname{Nor}(u)$ the vectors

$$
W_{i}=\left(\frac{1}{\sqrt{1+K_{i}(X, V)^{2}}} U_{i}, \frac{K_{i}(X, V)}{\sqrt{1+K_{i}(X, V)^{2}}} U_{i}\right), \quad i \in\{1, \ldots, n\}
$$

are an orthonormal basis of $\operatorname{Tan}^{n}\left(\mathcal{H}^{n}\llcorner\operatorname{Nor}(u),(X, V))\right.$. Furthermore, the unit vectors $U_{1}, \ldots, U_{n}$ represent a suitably chosen orthonormal basis of $V^{\perp}$ in $\mathbb{R}^{n+1}$. Of course, these vectors depend on the pair $(X, V)$ which is considered. More precisely, for $i \in\{1, \ldots, n\}, U_{i}$ is chosen to be a unit vector which corresponds to the curvature $K_{i}(X, V)$ of the unit normal bundle $\operatorname{Nor}\left(\mathfrak{X}_{u}\right)$ in the same way as, in Section 2 and for $i \in\{1, \ldots, d-1\}$, the unit vector $u_{i}(x, v)$ corresponds to the curvature $k_{i}(x, v)$ of the unit normal bundle $\operatorname{Nor}(X)$. In addition, it can be assumed that $\left(U_{1}, \ldots, U_{n}, V\right)$ is negatively oriented with respect to the standard basis $\left(E_{1}, \ldots, E_{n+1}\right)$ of $\mathbb{R}^{n+1}$. Finally, for $\mathcal{H}^{n}$ almost all $(X, V) \in \operatorname{Nor}(u)$, we set

$$
D_{i_{1} \ldots i_{j}}(X, V):=\frac{\left\langle V, E_{n+1}\right\rangle^{2}+\sum_{l=1}^{j}\left\langle U_{i_{l}}, E_{n+1}\right\rangle^{2}}{-\left\langle V, E_{n+1}\right\rangle} \geq-\left\langle V, E_{n+1}\right\rangle>0,
$$

provided that $j \in\{1, \ldots, n\}$ and $1 \leq i_{1}<\ldots<i_{j} \leq n$. For $j=0$ we set

$$
D_{i_{1} \ldots i_{0}}(X, V):=-\left\langle V, E_{n+1}\right\rangle .
$$

Now, we are in a position to present the main result of this section.

Theorem 3.1. Let $\Omega \subset \mathbb{R}^{n}$ be nonempty, open and convex, and let $u: \Omega \rightarrow \mathbb{R}$ be a convex function. Then, there exist positive measures $\Theta_{0}(u, \cdot), \ldots, \Theta_{n}(u, \cdot)$ on $\mathfrak{B}\left(\Omega \times \mathbb{R}^{n}\right)$ such that the Steiner formula

$$
\mathcal{H}^{n}\left(P_{\rho}(u, \eta)\right)=\sum_{j=0}^{n}\left(\begin{array}{l}
n \\
j
\end{array}\right) \rho^{j} \Theta_{n-j}(u, \eta)
$$

holds true for any $\eta \in \mathfrak{B}\left(\Omega \times \mathbb{R}^{n}\right)$ and $\rho \geq 0$. In addition, the coefficient measures $\Theta_{n-j}(u, \cdot), j \in\{0, \ldots, n\}$, can be represented by

$$
\begin{aligned}
& \left(\begin{array}{l}
n \\
j
\end{array}\right) \Theta_{n-j}(u, \eta)=\int_{\operatorname{Nor}(u) \cap \hat{\eta}}\left(-\frac{1}{\left\langle V, E_{n+1}\right\rangle}\right)^{j} \sum_{1 \leq i_{1}<\ldots<i_{j} \leq n} \\
& \times \frac{K_{i_{1}}(X, V) \cdots K_{i_{j}}(X, V)}{\prod_{i=1}^{n} \sqrt{1+K_{i}(X, V)^{2}}} D_{i_{1} \ldots i_{j}}(X, V) \mathrm{d} \mathcal{H}^{n}(X, V) .
\end{aligned}
$$

Proof. According to the preparatory considerations before Theorem [3.1, we have to calculate the approximate Jacobian ap $J_{n} F_{\rho}(X, V)$ of the mapping $F_{\rho}$ for $\mathcal{H}^{n}$ almost all $(X, V) \in \operatorname{Nor}(u)$. We shall adopt the previous notation. In the following, however, we shall often omit the argument $(X, V)$ of various functions, if there is 
no danger of ambiguity. Hence we get, for $\mathcal{H}^{n}$ almost all $(X, V) \in \operatorname{Nor}(u)$, that

$$
\begin{aligned}
\operatorname{ap} D F_{\rho}(X, V)\left(W_{i}\right)= & \frac{1}{\sqrt{1+K_{i}^{2}}}\left(U_{i}-\left\langle U_{i}, E_{n+1}\right\rangle E_{n+1}\right) \\
& -\frac{\rho}{\left\langle V, E_{n+1}\right\rangle} \frac{K_{i}}{\sqrt{1+K_{i}^{2}}}\left(U_{i}-\frac{\left\langle U_{i}, E_{n+1}\right\rangle}{\left\langle V, E_{n+1}\right\rangle} V\right) \\
= & \frac{1}{\sqrt{1+K_{i}^{2}}} A_{i}+\left(-\frac{1}{\left\langle V, E_{n+1}\right\rangle}\right) \rho \frac{K_{i}}{\sqrt{1+K_{i}^{2}}} B_{i},
\end{aligned}
$$

if

$$
A_{i}:=U_{i}-\left\langle U_{i}, E_{n+1}\right\rangle E_{n+1}
$$

and

$$
B_{i}:=U_{i}-\frac{\left\langle U_{i}, E_{n+1}\right\rangle}{\left\langle V, E_{n+1}\right\rangle} V
$$

for $i \in\{1, \ldots, n\}$. Note that $A_{i}, B_{i} \in E_{n+1}^{\perp}$. Now, the approximate Jacobian can be calculated according to

$$
\operatorname{ap} J_{n} F_{\rho}(X, V)=\left\|\bigwedge_{i=1}^{n} \operatorname{ap} D F_{\rho}(X, V)\left(W_{i}\right)\right\|
$$

and

$$
\begin{aligned}
\bigwedge_{i=1}^{n} \operatorname{ap} & D F_{\rho}(X, V)\left(W_{i}\right) \\
& =\bigwedge_{i=1}^{n}\left[\frac{1}{\sqrt{1+K_{i}^{2}}} A_{i}+\left(-\frac{1}{\left\langle V, E_{n+1}\right\rangle}\right) \rho \frac{K_{i}}{\sqrt{1+K_{i}^{2}}} B_{i}\right] \\
& =\sum_{j=0}^{n} \rho^{j}\left(-\frac{1}{\left\langle V, E_{n+1}\right\rangle}\right)^{j} \sum_{1 \leq i_{1}<\ldots<i_{j} \leq n} \frac{K_{i_{1}} \cdots K_{i_{j}}}{\prod_{i=1}^{n} \sqrt{1+K_{i}^{2}}} \bigwedge_{i_{1} \ldots i_{j}}(A, B) .
\end{aligned}
$$

The quantities $\bigwedge_{i_{1} \ldots i_{j}}(A, B)$ are defined by

$$
\bigwedge_{i_{1} \ldots i_{j}}(A, B):=C_{1} \wedge \ldots \wedge C_{n}
$$

where

$$
C_{i_{1}}:=B_{i_{1}}, \ldots, C_{i_{j}}:=B_{i_{j}}
$$

and

$$
C_{l}:=A_{l}, \quad l \in\{1, \ldots, n\} \backslash\left\{i_{1}, \ldots, i_{j}\right\},
$$

provided that $j \in\{1, \ldots, n\}$ and $1 \leq i_{1}<\ldots<i_{j} \leq n$. For $j=0$, however, we set

$$
\bigwedge_{i_{1} \ldots i_{0}}(A, B):=A_{1} \wedge \ldots \wedge A_{n} .
$$

Observe that

$$
\bigwedge_{i_{1} \ldots i_{j}}(A, B)=D_{i_{1} \ldots i_{j}}(A, B) E_{1} \wedge \ldots \wedge E_{n}
$$

with

$$
D_{i_{1} \ldots i_{j}}(A, B):=\operatorname{det}\left(C_{1}, \ldots, C_{n}\right),
$$


if $j \in\{1, \ldots, n\}$, and

$$
D_{i_{1} \ldots i_{0}}(A, B):=\operatorname{det}\left(A_{1}, \ldots, A_{n}\right) .
$$

Recall that $\left(E_{1}, \ldots, E_{n+1}\right)$ is the standard basis of $\mathbb{R}^{n+1}$, and $\left(E_{1}, \ldots, E_{n}\right)$ is supposed to be positively oriented with respect to the 'det' function on $E_{n+1}^{\perp}$. By some elementary linear algebra we can now deduce that

$$
\begin{aligned}
D_{i_{1} \ldots i_{j}}(A, B)= & \operatorname{det}\left(C_{1}, \ldots, C_{n}\right) \\
= & \operatorname{det}\left(C_{1}, \ldots, C_{n}, E_{n+1}\right) \\
= & \frac{\left\langle V, E_{n+1}\right\rangle^{2}+\sum_{l=1}^{j}\left\langle U_{i_{l}}, E_{n+1}\right\rangle^{2}}{\left\langle V, E_{n+1}\right\rangle} \operatorname{det}\left(U_{1}, \ldots, U_{n}, V\right) \\
= & \frac{\left\langle V, E_{n+1}\right\rangle^{2}+\sum_{l=1}^{j}\left\langle U_{i_{l}}, E_{n+1}\right\rangle^{2}}{-\left\langle V, E_{n+1}\right\rangle} \\
\geq & -\left\langle V, E_{n+1}\right\rangle>0,
\end{aligned}
$$

for $j \in\{0, \ldots, n\}$ and for any choice of the indices $1 \leq i_{1}<\ldots<i_{j} \leq n$.

But then we get

$$
\begin{aligned}
& \operatorname{ap} J_{n} F_{\rho}(X, V) \\
& =\sum_{j=0}^{n} \rho^{j}\left(-\frac{1}{\left\langle V, E_{n+1}\right\rangle}\right)^{j} \sum_{1 \leq i_{1}<\ldots<i_{j} \leq n} \frac{K_{i_{1}}(X, V) \cdots K_{i_{j}}(X, V)}{\prod_{i=1}^{n} \sqrt{1+K_{i}(X, V)^{2}}} D_{i_{1} \ldots i_{j}}(X, V),
\end{aligned}
$$

if we write $D_{i_{1} \ldots i_{j}}(X, V)$ instead of $D_{i_{1} \ldots i_{j}}(A, B)$, since this quantity basically is a function of $(X, V)$, cf. the preceding definitions (7) and (8). Moreover, we also used the fact that $K_{i}(X, V) \in[0, \infty]$, for $i \in\{1, \ldots, n\}$ and $\mathcal{H}^{n}$ almost all $(X, V) \in$ $\operatorname{Nor}\left(\mathfrak{X}_{u}\right)$.

Thus, from equations (6) and (11) we deduce the following Steiner formula:

$$
\begin{aligned}
& \mathcal{H}^{n}\left(P_{\rho}(u, \eta)\right) \\
& =\sum_{j=0}^{n}\left(\begin{array}{c}
n \\
j
\end{array}\right) \rho^{j} \frac{1}{\left(\begin{array}{c}
n \\
j
\end{array}\right)} \int_{\operatorname{Nor}(u) \cap \hat{\eta}}\left(-\frac{1}{\left\langle V, E_{n+1}\right\rangle}\right)^{j} \\
& \quad \times \sum_{1 \leq i_{1}<\ldots<i_{j} \leq n} \frac{K_{i_{1}}(X, V) \cdots K_{i_{j}}(X, V)}{\prod_{i=1}^{n} \sqrt{1+K_{i}(X, V)^{2}}} D_{i_{1} \ldots i_{j}}(X, V) \mathrm{d} \mathcal{H}^{n}(X, V) \\
& =: \sum_{j=0}^{n}\left(\begin{array}{c}
n \\
j
\end{array}\right) \rho^{j} \Theta_{n-j}(u, \eta),
\end{aligned}
$$

which holds true for any $\eta \in \mathfrak{B}\left(\Omega \times \mathbb{R}^{n}\right)$ and $\rho \geq 0$. The last line can serve as a defining equation for the coefficient curvature measures $\Theta_{n-j}(u, \cdot), j \in\{0, \ldots, n\}$, of the convex function $u$. These are measures on the $\sigma$-algebra $\mathfrak{B}\left(\Omega \times \mathbb{R}^{n}\right)$, which are nonnegative, since the integrand in the defining integral representation for these measures is a nonnegative function. 
Remark. A special case of the preceding Steiner formula implies that

$$
F_{j}(u, \beta)=\Theta_{n-j}\left(u, \beta \times \mathbb{R}^{n}\right),
$$

if $\beta \in \mathfrak{B}(\Omega)$. This establishes the connection with the measures $F_{j}(u, \cdot), j \in$ $\{0, \ldots, n\}$, which have previously been introduced in [6], under more restrictive assumptions on the associated convex function $u$, as measures on the $\sigma$-algebra $\mathfrak{B}(\Omega)$.

In the two special cases $j=0$ and $j=n$, the coefficient measures $\Theta_{n-j}(u, \cdot)$ will be discussed separately. Assume that $\eta \in \mathfrak{B}\left(\Omega \times \mathbb{R}^{n}\right)$.

First, let $j=0$. Since

$$
\operatorname{det}\left(A_{1}, \ldots, A_{n}\right)=-\left\langle V, E_{n+1}\right\rangle,
$$

we have

$$
\begin{aligned}
\mathcal{H}^{n}( & \{x \in \Omega: \exists p \in \partial u(x) \quad(x, p) \in \eta\}) \\
& =\mathcal{H}^{n}\left(P_{0}(u, \eta)\right) \\
& =\int_{\operatorname{Nor}(u) \cap \hat{\eta}}\left(-\left\langle V, E_{n+1}\right\rangle\right) \prod_{i=1}^{n} \frac{1}{\sqrt{1+K_{i}(X, V)^{2}}} \mathrm{~d} \mathcal{H}^{n}(X, V) \\
& =\Theta_{n}(u, \eta),
\end{aligned}
$$

and hence, in particular,

$$
\mathcal{H}^{n}(\beta)=\Theta_{n}\left(u, \beta \times \mathbb{R}^{n}\right)=F_{0}(u, \beta),
$$

if $\beta \in \mathfrak{B}(\Omega)$.

Now, let us investigate the case $j=n$. We have

$$
\operatorname{det}\left(B_{1}, \ldots, B_{n}\right)=\left(-\frac{1}{\left\langle V, E_{n+1}\right\rangle}\right),
$$

and thus we can write

$$
\Theta_{0}(u, \eta)=\int_{\operatorname{Nor}(u) \cap \hat{\eta}}\left(-\frac{1}{\left\langle V, E_{n+1}\right\rangle}\right)^{n+1} \prod_{i=1}^{n} \frac{K_{i}(X, V)}{\sqrt{1+K_{i}(X, V)^{2}}} \mathrm{~d} \mathcal{H}^{n}(X, V) .
$$

From the calculations in the proof of Theorem 3.1 one obtains for the approximate Jacobian of the map $\pi_{2} \circ \tilde{T}: \operatorname{Nor}(u) \rightarrow \mathbb{R}^{n}$ that

$$
\text { ap } J_{n}\left(\pi_{2} \circ \tilde{T}\right)(X, V)=\left(-\frac{1}{\left\langle V, E_{n+1}\right\rangle}\right)^{n+1} \prod_{i=1}^{n} \frac{K_{i}(X, V)}{\sqrt{1+K_{i}(X, V)^{2}}},
$$

for $\mathcal{H}^{n}$ almost all $(X, V) \in \operatorname{Nor}(u)$. Therefore,

$$
\begin{aligned}
\Theta_{0}(u, \eta) & =\int_{\mathbb{R}^{n}} \mathcal{H}^{0}\left(\hat{\eta} \cap\left(\pi_{2} \circ \tilde{T}\right)^{-1}(\{p\})\right) \mathrm{d} \mathcal{H}^{n}(p) \\
& =\int_{\mathbb{R}^{n}} \mathcal{H}^{0}(\{x \in \Omega:(x, p) \in \Gamma(u) \cap \eta\}) \mathrm{d} \mathcal{H}^{n}(p) \\
& =\mathcal{H}^{n}\left(\left\{p \in \mathbb{R}^{n}: \exists x \in \Omega \quad(x, p) \in \Gamma(u) \cap \eta\right\}\right) .
\end{aligned}
$$

As a special case we get that

$$
\Theta_{0}\left(u, \beta \times \mathbb{R}^{n}\right)=\mathcal{H}^{n}\left(\left\{p \in \mathbb{R}^{n}: \exists x \in \beta \quad p \in \partial u(x)\right\}\right),
$$


which is Theorem 3.1 from [6]. In the preceding argument we have used that for $\mathcal{H}^{n}$ almost all $p \in \mathbb{R}^{n}$ there is at most one $x \in \Omega$ such that $p \in \partial u(x)$. This follows from Theorem 2.2.9 in Schneider [14] and from the relation

$$
\begin{aligned}
& \left\{p \in \mathbb{R}^{n}: \operatorname{card}\left(\pi_{2}^{-1}(\{p\}) \cap \Gamma(u)\right) \geq 2\right\} \\
& \quad=H\left(\left\{V \in S^{n}: \operatorname{card}\left(\Pi_{2}^{-1}(\{V\}) \cap \operatorname{Nor}(u)\right) \geq 2\right\}\right),
\end{aligned}
$$

where $\Pi_{2}$ is defined by

$$
\Pi_{2}: \mathbb{R}^{n+1} \times \mathbb{R}^{n+1} \rightarrow \mathbb{R}^{n+1}, \quad(X, V) \mapsto V,
$$

and the map

$$
H: S^{n} \backslash E_{n+1}^{\perp} \rightarrow \mathbb{R}^{n}, \quad V \mapsto E_{n+1}-\left\langle V, E_{n+1}\right\rangle^{-1} V,
$$

is locally Lipschitzian.

Thus, to summarize, we have the following corollary.

Corollary 3.2. Let $\Omega \subset \mathbb{R}^{n}$ be open and convex, and let $u: \Omega \rightarrow \mathbb{R}$ be a convex function. Then, for any $\eta \in \mathfrak{B}\left(\Omega \times \mathbb{R}^{n}\right)$,

$$
\Theta_{n}(u, \eta)=\mathcal{H}^{n}\left(\left\{x \in \Omega: \pi_{1}^{-1}(\{x\}) \cap \eta \cap \Gamma(u) \neq \emptyset\right\}\right)
$$

and

$$
\Theta_{0}(u, \eta)=\mathcal{H}^{n}\left(\left\{p \in \mathbb{R}^{n}: \pi_{2}^{-1}(\{p\}) \cap \eta \cap \Gamma(u) \neq \emptyset\right\}\right) .
$$

\section{Weighted MEASURES OF SINGUlar POINTS}

In this second part of our treatment of convex functions, we shall investigate singularities of convex functions. Basically, we will continue to use the notation of Section 3. Recall from Section 1 that the set of $r$-singular points of the convex function $u: \Omega \rightarrow \mathbb{R}$ is defined by

$$
\Sigma^{r}(u):=\{x \in \Omega: \operatorname{dim} \partial u(x) \geq n-r\},
$$

if $r \in\{0, \ldots, n\}$. Note that for any fixed point $x \in \Omega$ there is a bi-Lipschitz transformation between $\partial u(x)$ and $N\left(\mathfrak{X}_{u},(x, u(x))\right) \cap S^{n}$ which is determined by

$$
p \mapsto \frac{1}{\sqrt{1+|p|^{2}}}(p,-1) .
$$

Moreover, the restriction of the map

$$
\Omega \rightarrow \operatorname{graph}(u) \subset \mathbb{R}^{n+1}, \quad x \mapsto(x, u(x)),
$$

to $\Sigma^{r}(u)$ yields a locally bi-Lipschitz correspondence between $\Sigma^{r}(u)$ and $\Sigma^{r}\left(\mathfrak{X}_{u}\right) \cap$ $\operatorname{graph}(u)$; cf. [11] for the notation concerning singular boundary points of convex sets. Hence, according to Lemma 3.1 in [11], the set $\Sigma^{r}(u)$ is a countably $r$-rectifiable Borel set in $\mathbb{R}^{n}$.

Theorem 4.1. Let $\Omega \subset \mathbb{R}^{n}$ be nonempty, open and convex, and let $u: \Omega \rightarrow \mathbb{R}$ be a convex function. Then, for $r \in\{0, \ldots, n\}$ and $\eta \in \mathfrak{B}\left(\Omega \times \mathbb{R}^{n}\right)$,

$$
\left(\begin{array}{l}
n \\
r
\end{array}\right) \Theta_{r}\left(u, \eta \cap\left(\Sigma^{r}(u) \times \mathbb{R}^{n}\right)\right)=\int_{\Sigma^{r}(u)} \mathcal{H}^{n-r}\left(\partial u(x) \cap \eta_{x}\right) \mathrm{d} \mathcal{H}^{r}(x),
$$

if $\eta_{x}:=\left\{p \in \mathbb{R}^{n}:(x, p) \in \eta\right\}$. 
Proof. To start with, let us define the set

$$
\operatorname{Nor}\left(\Sigma^{r}(u)\right):=\operatorname{Nor}(u) \cap\left(\Sigma^{r}\left(\mathfrak{X}_{u}\right) \times S^{n}\right),
$$

which is a countably $n$-rectifiable Borel subset of $\operatorname{Nor}\left(\mathfrak{X}_{u}\right) \subset \mathbb{R}^{n+1} \times \mathbb{R}^{n+1}$.

A first observation is that, for $\mathcal{H}^{n}$ almost all $(X, V) \in \operatorname{Nor}\left(\Sigma^{r}(u)\right)$, at least $(n-r)$ of the curvatures $K_{1}(X, V), \ldots, K_{n}(X, V)$ are equal to $\infty$. For a justification of this statement, see [11, proof of Theorem 3.2. Obviously, we can always assume that

$$
0 \leq K_{1}(X, V) \leq \ldots \leq K_{n}(X, V) \leq \infty .
$$

Subsequently, we will consider the locally Lipschitz map $G$ which is defined by

$$
G: \operatorname{Nor}\left(\Sigma^{r}(u)\right) \rightarrow \Sigma^{r}(u), \quad(X, V) \mapsto\left(\pi_{1} \circ \tilde{T}\right)(X, V) .
$$

First of all, assume that $r \in\{1, \ldots, n-1\}$. Using the calculations which are contained in the first part of the proof for Theorem 3.1 we obtain for $\mathcal{H}^{n}$ almost all $(X, V) \in \operatorname{Nor}\left(\Sigma^{r}(u)\right)$ that

$$
\operatorname{ap} J_{r} G(X, V)=\left\|\bigwedge_{i=1}^{r}\left(U_{i}-\left\langle U_{i}, E_{n+1}\right\rangle E_{n+1}\right)\right\| \prod_{i=1}^{r} \frac{1}{\sqrt{1+K_{i}(X, V)^{2}}} .
$$

This is proved by exactly the same arguments and with the same conventions as in the proof of Theorem 3.2 from [11. Furthermore, observe that

$$
\left\|\bigwedge_{i=1}^{r}\left(U_{i}-\left\langle U_{i}, E_{n+1}\right\rangle E_{n+1}\right)\right\|>0
$$

From the integral representation of $\Theta_{r}(u, \cdot)$, which is provided by Theorem 3.1 one can now deduce the relation

$$
\begin{aligned}
\left(\begin{array}{l}
n \\
r
\end{array}\right) \Theta_{r}\left(u, \eta \cap\left(\Sigma^{r}(u) \times \mathbb{R}^{n}\right)\right) & \\
=\int_{\operatorname{Nor}\left(\Sigma^{r}(u)\right) \cap \hat{\eta}} \operatorname{ap~} J_{r} G(X, V) & \left(-\frac{1}{\left\langle V, E_{n+1}\right\rangle}\right)^{n-r} \\
& \times \frac{D_{r+1 \ldots n}(X, V)}{\left\|\bigwedge_{i=1}^{r}\left(U_{i}-\left\langle U_{i}, E_{n+1}\right\rangle E_{n+1}\right)\right\|} \mathrm{d} \mathcal{H}^{n}(X, V)
\end{aligned}
$$

in the same way as equation (9) was deduced from equation (8) in the course of the proof of Theorem 3.2 in [11].

An application of Federer's coarea theorem then implies that

$$
\begin{aligned}
\left(\begin{array}{l}
n \\
r
\end{array}\right) \Theta_{r}\left(u, \eta \cap\left(\Sigma^{r}(u) \times \mathbb{R}^{n}\right)\right) & \\
=\int_{\Sigma^{r}(u)} \int_{G^{-1}(\{x\}) \cap \hat{\eta}} & \left(-\frac{1}{\left\langle V, E_{n+1}\right\rangle}\right)^{n-r} \\
& \times \frac{D_{r+1 \ldots n}(X, V)}{\left\|\bigwedge_{i=1}^{r}\left(U_{i}-\left\langle U_{i}, E_{n+1}\right\rangle E_{n+1}\right)\right\|} \mathrm{d} \mathcal{H}^{n-r}(X, V) \mathrm{d} \mathcal{H}^{r}(x) .
\end{aligned}
$$


Note that, for any fixed $x \in \Sigma^{r}(u)$,

$$
\begin{aligned}
& G^{-1}(\{x\}) \cap \hat{\eta} \\
& \quad=\left\{\left((x, u(x)), \frac{1}{\sqrt{1+|p|^{2}}}(p,-1)\right) \in \operatorname{Nor}\left(\Sigma^{r}(u)\right): p \in \mathbb{R}^{n},(x, p) \in \eta\right\} .
\end{aligned}
$$

The set

$$
\left\{x \in \Sigma^{r}(u): \operatorname{dim} \partial u(x)>n-r\right\}
$$

has $r$-dimensional Hausdorff measure zero. Therefore, in the following we only have to consider the case where $\operatorname{dim} \partial u(x)=n-r$.

Next, we define the bijective map

$$
f:\left\{\begin{array}{l}
G^{-1}(\{x\}) \rightarrow \partial u(x) \\
\left((x, u(x)), \frac{1}{\sqrt{1+|p|^{2}}}(p,-1)\right) \mapsto p
\end{array}\right.
$$

For $\mathcal{H}^{r}$ almost all $x \in \Sigma^{r}(u)$ the following holds true: for $\mathcal{H}^{n-r}$ almost all $(X, V) \in$ $G^{-1}(\{x\})$, the Jacobian $J_{n-r} f(X, V)$ of $f$ is equal to the Jacobian $J_{n-r} g(V)$ of the differentiable map $g$ which is defined by

$$
g:\left\{\begin{array}{l}
S^{n} \cap \operatorname{lin}\left\{U_{r+1}, \ldots, U_{n}, V\right\} \rightarrow \mathbb{R}^{n} \\
Y \mapsto E_{n+1}-\left\langle Y, E_{n+1}\right\rangle^{-1} Y
\end{array}\right.
$$

Once again it is important to emphasize that the unit vectors $U_{r+1}, \ldots, U_{n}$ depend on $(X, V) \in G^{-1}(\{x\})$. Nevertheless, for $\mathcal{H}^{r}$ almost all $x \in \Sigma^{r}(u)$ the following holds true: for $\mathcal{H}^{n-r}$ almost all $V \in N\left(\mathfrak{X}_{u},(x, u(x))\right) \cap S^{n}$, the $(n-r+1)$-dimensional vector space

$$
\operatorname{lin}\left\{U_{r+1}, \ldots, U_{n}, V\right\}=\operatorname{lin} N\left(\mathfrak{X}_{u},(x, u(x))\right)
$$

does not depend on the particular choice of $(X, V) \in G^{-1}(\{x\})$. Here it is assumed, as we always do, that the ordering of the vectors $U_{1}, \ldots, U_{n}$ is chosen properly.

A straightforward calculation then yields

$$
J_{n-r} g(V)=\left(-\frac{1}{\left\langle V, E_{n+1}\right\rangle}\right)^{n-r}\left\|\bigwedge_{i=r+1}^{n}\left(U_{i}-\frac{\left\langle U_{i}, E_{n+1}\right\rangle}{\left\langle V, E_{n+1}\right\rangle} V\right)\right\| .
$$

It is easy to verify by another elementary calculation that $\left\langle A_{i}, B_{j}\right\rangle=\delta_{i j}$ for all $i, j \in\{1, \ldots, n\}$; for definitions of these quantities see (9) and (10) in the proof of Theorem 3.1. From this observation we obtain

$$
\begin{aligned}
D_{r+1 \ldots n}(X, V) & =\left\|A_{1} \wedge \ldots \wedge A_{r} \wedge B_{r+1} \wedge \ldots \wedge B_{n}\right\| \\
& =\left\|A_{1} \wedge \ldots \wedge A_{r}\right\|\left\|B_{r+1} \wedge \ldots \wedge B_{n}\right\| .
\end{aligned}
$$


Hence, for $\mathcal{H}^{r}$ almost all $x \in \Sigma^{r}(u)$, we can infer that

$$
\begin{aligned}
& \int_{G^{-1}(\{x\}) \cap \hat{\eta}}\left(-\frac{1}{\left\langle V, E_{n+1}\right\rangle}\right)^{n-r} \frac{D_{r+1 \ldots n}(X, V)}{\left\|\bigwedge_{i=1}^{r}\left(U_{i}-\left\langle U_{i}, E_{n+1}\right\rangle E_{n+1}\right)\right\|} \mathrm{d} \mathcal{H}^{n-r}(X, V) \\
= & \int_{G^{-1}(\{x\})} \mathbf{1}_{\hat{\eta}}(X, V) J_{n-r} f(X, V) \mathrm{d} \mathcal{H}^{r}(X, V) \\
= & \int_{\partial u(x)} \mathbf{1}_{\eta}(x, p) \mathrm{d} \mathcal{H}^{r}(p) \\
= & \mathcal{H}^{r}\left(\partial u(x) \cap \eta_{x}\right) .
\end{aligned}
$$

A special case of the coarea formula can be used to justify the second equality. This immediately implies Theorem 4.1 in the case $r \in\{1, \ldots, n-1\}$.

Now, assume that $r=n$. But then the statement of the theorem is implied by Corollary [3.2, since

$$
\pi_{1}^{-1}(\{x\}) \cap \eta \cap \Gamma(u) \neq \emptyset \Leftrightarrow \partial u(x) \cap \eta_{x} \neq \emptyset
$$

holds for all $x \in \Omega$ and because $u$ is differentiable for $\mathcal{H}^{n}$ almost all $x \in \Sigma^{n}(u)=\Omega$. Alternatively, another proof follows from minor modifications of the argument for the case $r \in\{1, \ldots, n-1\}$.

Finally, we treat the case $r=0$. Then, $\Sigma^{0}(u)=\left\{x_{\iota}: \iota \in I\right\}$, where $I$ is at most countable. Note that

$$
K_{1}(X, V)=\ldots=K_{n}(X, V)=\infty,
$$

for $\mathcal{H}^{n}$ almost all $(X, V) \in \operatorname{Nor}\left(\Sigma^{0}(u)\right)$. Hence,

$$
\begin{aligned}
\Theta_{0} & \left(u, \eta \cap\left(\Sigma^{0}(u) \times \mathbb{R}^{n}\right)\right) \\
= & \int_{\operatorname{Nor}\left(\Sigma^{0}(u)\right) \cap \hat{\eta}}\left(-\frac{1}{\left\langle V, E_{n+1}\right\rangle}\right)^{n} \prod_{i=1}^{n} \frac{K_{i}(X, V)}{\sqrt{1+K_{i}(X, V)^{2}}} D_{1 \ldots n}(X, V) \mathrm{d} \mathcal{H}^{n}(X, V) \\
= & \sum_{\iota \in I}\left(-\frac{1}{\left\langle V, E_{n+1}\right\rangle}\right)^{n} D_{1 \ldots n}(X, V) \mathrm{d} \mathcal{H}^{n}(X, V) \\
= & \left.\sum_{\iota \in I} \mathcal{H}^{n}\left(\partial u\left(x_{\iota}\right\}\right) \cap \eta_{x_{\iota}}\right) \\
= & \int_{\Sigma^{0}(u)} \mathcal{H}^{n}\left(\partial u(x) \cap \eta_{x}\right) \mathrm{d} \mathcal{H}^{0}(x) .
\end{aligned}
$$

The third equality can be verified as in the proof of the corresponding statement in the case $r \in\{1, \ldots, n-1\}$.

This completes the proof in all cases.

The following special case of Theorem 4.1 will be essential for the proof of Theorem 6.2 below. 
Corollary 4.2. Let $\Omega \subset \mathbb{R}^{n}$ be nonempty, open and convex, and let $u: \Omega \rightarrow \mathbb{R}$ be a convex function. Then,

$$
\begin{gathered}
\left(\begin{array}{l}
n \\
r
\end{array}\right) \Theta_{r}\left(u,\left(\Sigma^{r}(u) \cap \beta\right) \times \mathbb{R}^{n}\right)=\left(\begin{array}{c}
n \\
n-r
\end{array}\right) F_{n-r}\left(u, \Sigma^{r}(u) \cap \beta\right) \\
=\int_{\Sigma^{r}(u) \cap \beta} \mathcal{H}^{n-r}(\partial u(x)) \mathrm{d} \mathcal{H}^{r}(x),
\end{gathered}
$$

for all $r \in\{0, \ldots, n\}$ and $\beta \in \mathfrak{B}(\Omega)$.

\section{SEmi-COnVEX FunCtions}

In the following, the corresponding approach for semi-convex functions will be outlined. The method is strictly analogous to the one of Section 3, if some results for sets of positive reach, or, more generally, sets with the unique footpoint property, are used. We start with a definition of semi-convexity which is appropriate for our purpose; see also Bangert [3], Fu [10, and Remark 2 after Theorem [5.2 below. If $\Omega \subset \mathbb{R}^{n}$ is a nonempty open set, then we shall write $U \subset \subset \Omega$ if and only if $U$ is a nonempty, bounded, open and convex set such that $\bar{U} \subset \Omega$.

Definition. Let $\Omega \subset \mathbb{R}^{n}$ be nonempty, open and convex. A function $u: \Omega \rightarrow \mathbb{R}$ is called semi-convex, if for each set $U$ with $U \subset \subset \Omega$ there is a nonnegative constant $C$ such that the function

$$
k(x):=u(x)+\frac{C}{2}|x|^{2}, \quad x \in U,
$$

is convex. The smallest such constant is denoted by $S C(u, U)$.

A few remarks are in order. Let $\Omega, U$, and $u$ be given as in the preceding definition. Then, in particular, the function

$$
x \mapsto u(x)+\frac{S C(u, U)}{2}|x|^{2}, \quad x \in U,
$$

is convex. This is equivalent to the condition that

$$
u((1-t) x+t y)-(1-t) u(x)-t u(y) \leq \frac{S C(u, U)}{2}(1-t) t|x-y|^{2},
$$

for all $t \in[0,1]$ and $x, y \in U$. The definition of semi-convexity immediately implies that the restriction of $u$ to any compact subset of $\Omega$ is a Lipschitz map. Therefore, we can adopt the definition of the (generalized) subgradient $\partial u(x)$ of $u$ at $x \in \Omega$ from Clarke [4 p. 27].

From this definition one can deduce that, for any $x \in \Omega, \partial u(x)$ is convex and nonempty. Moreover, for $x \in U$, the subgradient $\partial u(x)$ can be calculated according to

$$
\partial u(x)=\partial k(x)-S C(u, U) x,
$$

where

$$
k(y):=u(y)+\frac{S C(u, U)}{2}|y|^{2}, \quad y \in U,
$$

is a convex function. This follows from Clarke [4, pp. 38-40]. In particular, $\partial k(x)$ coincides with the subdifferential in the sense of convexity. (Note that by a slight abuse of notation, we sometimes write $z$ instead of $\{z\}$, if $z \in \mathbb{R}^{n}$.) 
Therefore, if $\eta \in \mathfrak{B}\left(\Omega \times \mathbb{R}^{n}\right)$ and $\rho \geq 0$, then formally the graph $\Gamma(u)$ of the subgradient map $\partial u$ and the local parallel sets $P_{\rho}(u, \eta)$ can be defined in the same way as in the convex case.

After these preparations, we can state a straightforward extension of Proposition 2.2 from 6 .

Lemma 5.1. Let $\Omega \subset \mathbb{R}^{n}$ be nonempty, open and convex, and let $u: \Omega \rightarrow \mathbb{R}$ be a semi-convex function. Assume that $U \subset \subset \Omega$, and let $\rho \in\left[0, S C(u, U)^{-1}\right)$. Then, for each $z \in P_{\rho}\left(u, U \times \mathbb{R}^{n}\right)$, there is a unique point $p_{\rho}(z) \in U$ such that $z=p_{\rho}(z)+\rho v$ holds for some $v \in \partial u\left(p_{\rho}(z)\right)$. Moreover, the mapping

$$
p_{\rho}: P_{\rho}\left(u, U \times \mathbb{R}^{n}\right) \rightarrow U, \quad z \mapsto p_{\rho}(z),
$$

is Lipschitz continuous with Lipschitz constant $(1-\rho S C(u, U))^{-1}$.

Proof. Set $C:=S C(u, U)$ and define

$$
k(x):=u(x)+\frac{C}{2}|x|^{2}, \quad x \in U .
$$

The preceding remarks yield that

$$
\partial k(x)=\partial u(x)+C x, \quad x \in U .
$$

Now, choose $x, x^{\prime} \in U$ and $p \in \partial u(x), p^{\prime} \in \partial u\left(x^{\prime}\right)$. Hence we get $\bar{p}:=p+C x \in \partial k(x)$ and $\bar{p}^{\prime}:=p^{\prime}+C x^{\prime} \in \partial k\left(x^{\prime}\right)$, and thus

$$
\begin{aligned}
\left|x+\rho p-\left(x^{\prime}+\rho p^{\prime}\right)\right| & =(1-\rho C)\left|x+\frac{\rho}{1-\rho C} \bar{p}-\left(x^{\prime}+\frac{\rho}{1-\rho C} \bar{p}^{\prime}\right)\right| \\
& \geq(1-\rho C)\left|x-x^{\prime}\right| .
\end{aligned}
$$

An application of Proposition 2.2 from [6] justifies the last inequality.

Example. Define $U(a, r):=\left\{x \in \mathbb{R}^{n}:|x-a|<r\right\}$, if $a \in \mathbb{R}^{n}$ and $r \geq 0$. Let $\Omega:=U(o, 1)$ and $u(x):=(-1 / 2)|x|^{2}, x \in \Omega$. Then we have $S C(u, U)=1$, for all $U \subset \subset \Omega$, and $P_{\rho}\left(u, \Omega \times \mathbb{R}^{n}\right)=U(o, 1-\rho)$, if $\rho \in[0,1)$. Moreover, the map $p_{\rho}$ is given by

$$
p_{\rho}: U(o, 1-\rho) \rightarrow U(o, 1), \quad z \mapsto(1-\rho)^{-1} z,
$$

for $\rho \in[0,1)$. This trivial example shows that Lemma 5.1 cannot be improved.

Our next purpose is to define the generalized unit normal bundle Nor $(u)$ of the semi-convex function $u: \Omega \rightarrow \mathbb{R}$, and to describe its properties. One way to do this would be to define $\operatorname{Nor}(u):=T(\Gamma(u))$, cf. Section 3 , and to deduce rectifiability properties of $\operatorname{Nor}(u)$ from corresponding properties of $\Gamma(u)$. In order to be able to define generalized curvatures on $\operatorname{Nor}(u)$, however, we will proceed in a different manner. First, choose $U \subset \subset \Omega$ arbitrarily. Then we have that $\operatorname{Lip}(u \mid U)<\infty$ and $S C(u, U)<\infty$. According to Proposition 1.7 of $\mathrm{Fu}$ [10], there is a mapping $u(U): \mathbb{R}^{n} \rightarrow \mathbb{R}$ such that

$$
u(U)|U=u| U, \quad \operatorname{Lip}(u(U))<\infty
$$

and

$$
\sup \left\{S C(u(U), W): W \subset \subset \mathbb{R}^{n}\right\}=S C(u, U)<\infty .
$$

Set $\mathfrak{X}_{u(U)}:=\operatorname{epi}(u(U))$ and $\mathfrak{X}_{u}:=\overline{\text { epi }(u)}$, both of which are closed subsets of $\mathbb{R}^{n+1}$.

Theorem 2.3 of $\mathrm{Fu}$ [10] now implies that

$$
\operatorname{reach}\left(\mathfrak{X}_{u(U)}\right) \geq S C(u, U)^{-1}>0,
$$


and hence Nor $\left(\mathfrak{X}_{u(U)}\right)$ is a countably $n$-rectifiable Borel set in $\mathbb{R}^{n+1} \times \mathbb{R}^{n+1}$. Moreover, we then also get that

$$
\operatorname{reach}\left(\mathfrak{X}_{u},(x, u(x))\right)>0, \quad x \in U,
$$

since $U$ is open. This observation, Theorem 4.8 (12) in Federer [8], and Lemma 2.9 in $\mathrm{Fu}$ [10] yield that

$$
\begin{aligned}
\operatorname{Nor} & \left(\mathfrak{X}_{u},(x, u(x))\right) \cap S^{n} \\
& =\operatorname{Nor}\left(\mathfrak{X}_{u(U)},(x, u(x))\right) \cap S^{n} \\
& =\left\{\frac{1}{\sqrt{1+|p|^{2}}}(p,-1) \in \mathbb{R}^{n+1}: p \in \partial u(x)\right\},
\end{aligned}
$$

if $x \in U$ is arbitrarily chosen. Then we define

$$
\begin{aligned}
\operatorname{Nor}(u) & :=\bigcup_{U \subset \subset \Omega} \bigcup_{x \in U}\left(\operatorname{Nor}\left(\mathfrak{X}_{u(U)},(x, u(x))\right) \cap S^{n}\right) \\
& =\bigcup_{i=1}^{\infty}\left(\operatorname{Nor}\left(\mathfrak{X}_{u\left(U_{i}\right)}\right) \cap\left(\operatorname{graph}\left(u \mid U_{i}\right) \times S^{n}\right)\right),
\end{aligned}
$$

where $\left(U_{i}\right)_{i \in \mathbb{N}}$ is a sequence of sets such that $U_{i} \subset \subset \Omega$ for all $i \in \mathbb{N}$ and $\bigcup_{i=1}^{\infty} U_{i}=\Omega$.

Therefore, Nor $(u)$ is a countably $n$-rectifiable Borel set. In addition, the functions $T$ and $\tilde{T}$, which are defined as in Section 3, again are mutually inverse homeomorphisms, and locally they are Lipschitz maps. Thus, in particular, $\Gamma(u)$ is a countably $n$-rectifiable Borel set. Moreover, note that

$$
\operatorname{Nor}(u) \cap\left(\operatorname{graph}(u \mid U) \times S^{n}\right)=T\left(\Gamma(u) \cap\left(U \times \mathbb{R}^{n}\right)\right),
$$

for all $U \subset \subset \Omega$.

Let $U \subset \subset \Omega, \eta \in \mathfrak{B}\left(U \times \mathbb{R}^{n}\right)$, and define $\hat{\eta}:=T(\eta \cap \Gamma(u))$ and $F_{\rho}$ as in Section 3 . If not explicitly stated otherwise, then we adopt all conventions and definitions from Section 3. Thus we get $P_{\rho}(u, \eta)=F_{\rho}(\hat{\eta}) \subset \mathbb{R}^{n}$. Moreover, $F_{\rho} \mid T\left(\Gamma(u) \cap\left(U \times \mathbb{R}^{n}\right)\right)$ is an injective map provided that $0<\rho<S C(u, U)^{-1}$. This follows from Lemma 5.1. Hence, in particular, we obtain that $P_{\rho}(u, \eta) \in \mathfrak{B}\left(\mathbb{R}^{n}\right)$.

Again let $U \subset \subset \Omega$ be arbitrarily chosen. The results of Section 2 show that generalized curvatures $K_{1}(X, V), \ldots, K_{n}(X, V)$ are defined for $\mathcal{H}^{n}$ almost all $(X, V) \in$ $\operatorname{Nor}\left(\mathcal{X}_{u(U)}\right)$. From the preceding discussion and the local nature of these curvatures it can be seen that, for $\mathcal{H}^{n}$ almost all $(X, V) \in T\left(\Gamma(u) \cap\left(U \times \mathbb{R}^{n}\right)\right)$, these curvatures $K_{1}(X, V), \ldots, K_{n}(X, V)$ do not depend on the particular extension $u(U)$ of $u$. Also, for $\mathcal{H}^{n}$ almost all $(X, V) \in T\left(\Gamma(u) \cap\left(U \times \mathbb{R}^{n}\right)\right)$, a similar remark applies to the orthonormal basis $\left(U_{1}, \ldots, U_{n}\right)$ of $V^{\perp}$, which is connected with these curvatures, and hence also to the quantities $D_{i_{1} \ldots i_{j}}(X, V)$ which are defined as in Section 3. Furthermore, since $U \subset \subset \Omega$ was arbitrarily chosen, all these quantities are functions which depend only on $\operatorname{Nor}(u)$.

Now we are prepared to state a local Steiner formula for semi-convex functions.

Theorem 5.2. Let $\Omega \subset \mathbb{R}^{n}$ be nonempty, open and convex, and let $u: \Omega \rightarrow \mathbb{R}$ be a semi-convex function. Assume that $U \subset \subset \Omega$. Then, there are signed measures $\Theta_{0}(u, \cdot), \ldots, \Theta_{n}(u, \cdot)$ on $\mathfrak{B}\left(U \times \mathbb{R}^{n}\right)$ such that, for any $\eta \in \mathfrak{B}\left(U \times \mathbb{R}^{n}\right)$ and $\rho \in$ $\left[0, S C(u, U)^{-1}\right)$, the Steiner formula

$$
\mathcal{H}^{n}\left(P_{\rho}(u, \eta)\right)=\sum_{j=0}^{n}\left(\begin{array}{l}
n \\
j
\end{array}\right) \rho^{j} \Theta_{n-j}(u, \eta)
$$


holds true. In addition, the coefficient measures $\Theta_{n-j}(u, \cdot)$ can be represented by

$$
\begin{aligned}
\left(\begin{array}{c}
n \\
j
\end{array}\right) \Theta_{n-j}(u, \eta)= & \int_{\operatorname{Nor}(u) \cap \hat{\eta}} \\
& \left(-\frac{1}{\left\langle V, E_{n+1}\right\rangle}\right)^{j} \sum_{1 \leq i_{1}<\ldots<i_{j} \leq n} \\
\times & \frac{K_{i_{1}}(X, V) \cdots K_{i_{j}}(X, V)}{\prod_{i=1}^{n} \sqrt{1+K_{i}(X, V)^{2}}} D_{i_{1} \ldots i_{j}}(X, V) \mathrm{d} \mathcal{H}^{n}(X, V),
\end{aligned}
$$

if $j \in\{0, \ldots, n\}$ and $\eta \in \mathfrak{B}\left(U \times \mathbb{R}^{n}\right)$.

Proof. The proof is essentially the same as the one for Theorem 3.1 The only additional complication which arises consists in verifying that, for $\mathcal{H}^{n}$ almost all $(X, V) \in T\left(\Gamma(u) \cap\left(U \times \mathbb{R}^{n}\right)\right)$ and $\rho \in\left(0, S C(u, U)^{-1}\right)$, we have

$$
\begin{aligned}
& h(\rho, X, V) \\
& \quad:=\operatorname{det}\left(\frac{1}{\sqrt{1+K_{i}^{2}}} A_{i}+\left(-\frac{1}{\left\langle V, E_{n+1}\right\rangle}\right) \rho \frac{K_{i}}{\sqrt{1+K_{i}^{2}}} B_{i}, i=1, \ldots, n\right)>0,
\end{aligned}
$$

provided that all bases and determinants are oriented in the same way as in the proof of Theorem 3.1. Of course, $A_{i}, B_{i}$, and $K_{i}, i \in\{1, \ldots, n\}$, are functions of $(X, V)$. Since the epigraph of a semi-convex function need not be a convex set, the curvature functions associated with $\operatorname{Nor}(u)$ can be negative. This is why, compared with the proof in the convex case, an additional argument is required.

In order to establish that relation (12) holds true, consider the maps

$$
F_{\rho}: T\left(\Gamma(u) \cap\left(U \times \mathbb{R}^{n}\right)\right) \rightarrow P_{\rho}\left(u, U \times \mathbb{R}^{n}\right)
$$

and

$$
G_{\rho}:\left\{\begin{array}{l}
P_{\rho}\left(u, U \times \mathbb{R}^{n}\right) \rightarrow T\left(\Gamma(u) \cap\left(U \times \mathbb{R}^{n}\right)\right), \\
z \mapsto T\left(p_{\rho}(z), \frac{1}{\rho}\left(z-p_{\rho}(z)\right)\right),
\end{array}\right.
$$

for some fixed $\rho \in\left(0, S C(u, U)^{-1}\right)$. Since $F_{\rho}, G_{\rho}$ are mutually inverse homeomorphisms which locally are Lipschitz maps, we obtain that, for $\mathcal{H}^{n}$ almost all $(X, V) \in T\left(\Gamma(u) \cap\left(U \times \mathbb{R}^{n}\right)\right)$, the condition $h(\rho, X, V) \neq 0$ is fulfilled. Note that the set of $\mathcal{H}^{n}$ measure zero which has to be excluded can be chosen independently of $\rho \in\left(0, S C(u, U)^{-1}\right)$; cf. Zähle [16, p. 560] and Federer [8, Lemma 5.1]. Hence, for $\mathcal{H}^{n}$ almost all $(X, V) \in T\left(\Gamma(u) \cap\left(U \times \mathbb{R}^{n}\right)\right)$, we get that

$$
\operatorname{sgn} h(\rho, X, V)=\lim _{\rho \downarrow 0} \operatorname{sgn} h(\rho, X, V) .
$$

Suppose that precisely $(n-r)$ of the curvatures $K_{1}(X, V), \ldots, K_{n}(X, V)$ are infinite, say $K_{r+1}(X, V)=\ldots=K_{n}(X, V)=\infty$, for simplicity, where $r \in\{0, \ldots, n\}$. But then

$$
\begin{aligned}
h(\rho, X, V)=\prod_{i=1}^{r} \frac{1}{\sqrt{1+K_{i}(X, V)^{2}}} & \left(-\frac{1}{\left\langle V, E_{n+1}\right\rangle}\right)^{n-r} \\
& \times \rho^{n-r} \operatorname{det}\left(A_{1}, \ldots, A_{r}, B_{r+1}, \ldots, B_{n}\right)+o\left(\rho^{n-r}\right),
\end{aligned}
$$

and thus

$$
\operatorname{sgn} h(\rho, X, V)=\operatorname{sgn} \operatorname{det}\left(A_{1}, \ldots, A_{r}, B_{r+1}, \ldots, B_{n}\right),
$$


if $\rho \in\left(0, S C(u, U)^{-1}\right)$ is sufficiently small. In Section 3, it was shown that the righthand side of equation (13) is equal to 1 , and this proves the initial statement.

Remarks. 1. Note that the representation of the coefficient measures provided by Theorem 5.2 is independent of the particular choice of the set $U \subset \subset \Omega$.

2 . Let $\Omega \subset \mathbb{R}^{n}$ be nonempty and open (and not necessarily convex). Then it is possible to work with a more general notion of semi-convexity by defining $u: \Omega \rightarrow \mathbb{R}$ to be a semi-convex function if and only if for every $x \in \Omega$ there are a nonempty open convex neighbourhood $U_{x} \subset \Omega$ and a finite positive constant $C$ such that the function

$$
k(y):=u(y)+\frac{C}{2}|y|^{2}, \quad y \in U_{x},
$$

is convex. This is equivalent to demanding that $u$ be a lower- $C^{2}$ function, see Rockafellar 13, p. 374]. Under this weaker assumption on a function $u$, coefficient measures $\Theta_{j}(u, \cdot)$ can still be defined by the representation given in Theorem 5.2. Then, however, the Steiner formula is only true for bounded Borel sets $\eta$ which are contained in suitable neighbourhoods $U_{x} \times S^{n}$ with $x \in \Omega$. On the other hand, Theorem 5.3 below remains true in full generality.

The analogue of Theorem 4.1 remains valid in the setting of semi-convex functions. Since the proof carries over without essential changes, we merely state the result.

Theorem 5.3. Let $\Omega \subset \mathbb{R}^{n}$ be nonempty, open and convex, and let $u: \Omega \rightarrow \mathbb{R}$ be a semi-convex function. Then, we have

$$
\left(\begin{array}{l}
n \\
r
\end{array}\right) \Theta_{r}\left(u, \eta \cap\left(\Sigma^{r}(u) \times \mathbb{R}^{n}\right)\right)=\int_{\Sigma^{r}(u)} \mathcal{H}^{n-r}\left(\Gamma(u) \cap \eta_{x}\right) \mathrm{d} \mathcal{H}^{r}(x),
$$

for $r \in\{0, \ldots, n\}$ and $\eta \in \mathfrak{B}\left(\Omega \times \mathbb{R}^{n}\right)$.

Remark. The proof of Theorem 5.3 shows that the restriction of the measure $\Theta_{r}(u, \cdot)$ to the set $\left(\Sigma^{r}(u) \cap U\right) \times \mathbb{R}^{n}$ is nonnegative for all $U \subset \subset \Omega$. Therefore, the statement of Theorem 5.3 still makes sense, if $\eta \in \mathfrak{B}\left(\Omega \times \mathbb{R}^{n}\right)$.

\section{Sharp inequalities For Weighted MeAsures}

In Theorem 2 of $\left[6\right.$, an upper bound is established for the values $F_{r}(u, \Omega)$, $r \in\{0, \ldots, n\}$, if $\Omega$ is a sublevel set of $u$. There, the overall assumption is that $\Omega$ is a nonempty open bounded convex subset of $\mathbb{R}^{n}$ and $u: \Omega \rightarrow \mathbb{R}$ is a convex Lipschitz function. The measures $F_{r}(u, \cdot)$ are defined on the $\sigma$-algebra $\mathfrak{B}(\Omega)$, and they are related to the measures $\Theta_{r}(u, \cdot)$ of the present paper by the relation

$$
F_{r}(u, \beta)=\Theta_{n-r}\left(u, \beta \times \mathbb{R}^{n}\right),
$$

which holds for $\beta \in \mathfrak{B}(\Omega)$ and $r \in\{0, \ldots, n\}$. In Theorem 6.2 below we prove that Theorem 2 of [6] holds without the assumption that $\Omega$ is a sublevel set. The proof of this result was made possible by an inequality proved by Trudinger, see inequality (4.12) in [15], here stated as a lemma.

Let $u \in C^{\infty}\left(\mathbb{R}^{n}\right)$ be a convex Lipschitz function, and let $\Omega$ be a nonempty open bounded convex subset of $\mathbb{R}^{n}$ whose boundary is of class $C^{\infty}$. Then, for every $i \in\{0, \ldots, n\}$, let $S_{i}(u, x)$ denote the $i$-th elementary symmetric function of the eigenvalues of the Hessian matrix of $u$. Furthermore, for every $j \in\{0, \ldots, n-1\}$, let 
$H_{j}(\bar{\Omega}, y)$ denote the $j$-th elementary symmetric function of the principal curvatures of $\partial \Omega$ at $y$, where $\bar{\Omega}$ denotes the topological closure of $\Omega$.

Lemma 6.1 (Trudinger). In the above notation, for every $i \in\{1, \ldots, n\}$ the inequality

$$
i \int_{\Omega} S_{i}(u, x) \mathrm{d} \mathcal{H}^{n}(x) \leq L^{i} \int_{\partial \Omega} H_{i-1}(\bar{\Omega}, y) \mathrm{d} \mathcal{H}^{n-1}(y)
$$

holds, where $L$ denotes the Lipschitz constant of $u$ in $\Omega$.

Theorem 6.2 contains the promised estimates from above for the values $F_{r}(u, \Omega)$, $r \in\{0, \ldots, n\}$. The inequalities involve the Lipschitz constant of the convex function $u$ and the quermassintegrals $W_{r}(\bar{\Omega}), r \in\{0, \ldots, n\}$, of the convex body $\bar{\Omega}$ which is associated with the domain $\Omega$ of $u$. See Schneider [14 for a definition of these quermassintegrals.

Theorem 6.2. Let u be a convex Lipschitz function, defined on a nonempty open bounded convex set $\Omega \subset \mathbb{R}^{n}$. Denote by $L$ the Lipschitz constant of $u$ in $\Omega$. If $\Omega^{\prime}$ is an arbitrary compact convex subset of $\Omega$, then

$$
F_{r}\left(u, \Omega^{\prime}\right) \leq L^{r} W_{r}\left(\Omega^{\prime}\right),
$$

and, in particular,

$$
F_{r}(u, \Omega) \leq L^{r} W_{r}(\bar{\Omega})
$$

for every $r \in\{0, \ldots, n\}$.

Proof. First of all, note that the second inequality is an immediate consequence of the first inequality. In fact, there obviously exists an increasing sequence of convex bodies $\Omega_{l}^{\prime} \subset \Omega, l \in \mathbb{N}$, such that

$$
\bigcup_{l \in \mathbb{N}} \Omega_{l}^{\prime}=\Omega
$$

To each of the bodies $\Omega_{l}^{\prime}$ the first inequality can be applied, and hence, for each $r \in\{0, \ldots, n\}$,

$$
\begin{aligned}
F_{r}(u, \Omega) & =\lim _{l \rightarrow \infty} F_{r}\left(u, \Omega_{l}^{\prime}\right) \\
& \leq \lim _{l \rightarrow \infty} L^{r} W_{r}\left(\Omega_{l}^{\prime}\right) \\
& \leq L^{r} W_{r}(\bar{\Omega}),
\end{aligned}
$$

since the quermassintegrals are monotone functionals. Therefore, it is sufficient to consider the first inequality.

For the case $r=0$, recall that $F_{0}\left(u, \Omega^{\prime}\right)=\mathcal{H}^{n}\left(\Omega^{\prime}\right)=W_{0}\left(\Omega^{\prime}\right)$. Hence the theorem is true in this case.

Now let us consider the case that $r \in\{1, \ldots, n\}$. Let $\Omega^{\prime} \subset \Omega$ be fixed. Then there are open convex sets $\Omega_{1}, \Omega_{2}$ such that

$$
\Omega^{\prime} \subset \Omega_{1} \subset \bar{\Omega}_{1} \subset \Omega_{2} \subset \bar{\Omega}_{2} \subset \Omega .
$$

According to Lemma 2.3 in [6] there is a convex Lipschitz function $w: \mathbb{R}^{n} \rightarrow \mathbb{R}$ such that $u\left|\Omega_{2}=w\right| \Omega_{2}$. In particular, this implies that

$$
\operatorname{Lip}\left(w \mid \Omega_{2}\right)=\operatorname{Lip}\left(u \mid \Omega_{2}\right) \leq L .
$$


Furthermore, it is shown in Lemma 2.4 of [6] that the function $w$, which is constructed in a special way, can be approximated by a sequence of convex Lipschitz functions $w_{k} \in C^{\infty}\left(\mathbb{R}^{n}\right), k \in \mathbb{N}$, such that

$$
\operatorname{Lip}\left(w_{k} \mid \Omega_{1}\right) \leq \operatorname{Lip}\left(w \mid \Omega_{2}\right) \leq L,
$$

for all $k \in \mathbb{N}, w_{k} \rightarrow w$ uniformly on compact sets for $k \rightarrow \infty$, and such that the sequence $F_{r}\left(w_{k}, \cdot\right)$ converges weakly to $F_{r}(w, \cdot)$ on $\mathfrak{B}(\Omega)$ for $k \rightarrow \infty$. See Ash [2] for the notion of weak convergence of measures. The last statement follows from the proof of Theorem 1 in [6].

For an arbitrary fixed $\epsilon>0$, there exists an open convex set $\Omega_{\epsilon}$ with boundary of class $C^{\infty}$ such that $\Omega^{\prime} \subset \Omega_{\epsilon} \subset \Omega_{1}$ and

$$
W_{r}\left(\bar{\Omega}_{\epsilon}\right) \leq W_{r}\left(\Omega^{\prime}\right)+\epsilon .
$$

This fact is true, since every convex body can be approximated in the Hausdorff metric by a sequence of smooth convex bodies (see Theorem 3.3.1 in Schneider 14]), and the quermassintegrals are continuous with respect to this metric.

By the weak convergence and nonnegativity of the measures which we consider, we have

$$
F_{r}\left(w, \Omega^{\prime}\right) \leq F_{r}\left(w, \Omega_{\epsilon}\right) \leq \liminf _{k \rightarrow \infty} F_{r}\left(w_{k}, \Omega_{\epsilon}\right) .
$$

Since $w_{k} \in C^{\infty}\left(\mathbb{R}^{n}\right)$, Proposition 2.1 from [6] implies that $F_{r}\left(w_{k}, \cdot\right)$ admits the representation

$$
\left(\begin{array}{l}
n \\
r
\end{array}\right) F_{r}\left(w_{k}, \beta\right)=\int_{\beta} S_{r}\left(w_{k}, x\right) \mathrm{d} \mathcal{H}^{n}(x),
$$

for any $\beta \in \mathfrak{B}\left(\mathbb{R}^{n}\right)$.

On the other hand, we obtain from formula (4.2.28) in 14 that

$$
\left(\begin{array}{l}
n-1 \\
r-1
\end{array}\right) W_{r}\left(\bar{\Omega}_{\epsilon}\right)=\frac{1}{n} \int_{\partial \Omega_{\epsilon}} H_{r-1}\left(\bar{\Omega}_{\epsilon}, x\right) \mathrm{d} \mathcal{H}^{n-1}(x),
$$

since $\partial \Omega_{\epsilon}$ was assumed to be sufficiently smooth.

Thus, equation (16), Lemma 6.1, equation (17), and (14) imply

$$
\begin{aligned}
\left(\begin{array}{c}
n \\
r
\end{array}\right) \liminf _{k \rightarrow \infty} F_{r}\left(w_{k}, \Omega_{\epsilon}\right) & =\liminf _{k \rightarrow \infty} \int_{\Omega_{\epsilon}} S_{r}\left(w_{k}, x\right) \mathrm{d} \mathcal{H}^{n}(x) \\
& \leq \frac{L^{r}}{r} \int_{\partial \Omega_{\epsilon}} H_{r-1}\left(\bar{\Omega}_{\epsilon}, x\right) \mathrm{d} \mathcal{H}^{n-1}(x) \\
& =\left(\begin{array}{c}
n \\
r
\end{array}\right) L^{r} W_{r}\left(\bar{\Omega}_{\epsilon}\right) \\
& \leq\left(\begin{array}{c}
n \\
r
\end{array}\right) L^{r}\left(W_{r}\left(\Omega^{\prime}\right)+\epsilon\right) .
\end{aligned}
$$

From (15) and (18) we now obtain

$$
F_{r}\left(w, \Omega^{\prime}\right) \leq L^{r}\left(W_{r}\left(\Omega^{\prime}\right)+\epsilon\right) .
$$

This proves the theorem, since $\epsilon>0$ was arbitrarily chosen. 
The following theorem provides upper bounds for suitably weighted Hausdorff measures of sets of singular points for a given convex Lipschitz function. It is a straightforward consequence of Corollary 4.2, Theorem 6.2, and the fact that the measures $F_{r}(u, \cdot)$ are nonnegative, if $u$ is a convex function.

Theorem 6.3. Let $u$ be a convex Lipschitz function, defined on a nonempty open bounded convex set $\Omega \subset \mathbb{R}^{n}$. Denote by $L$ the Lipschitz constant of $u$ in $\Omega$. Then, for every $r \in\{0, \ldots, n\}$, the following inequality holds true:

$$
\int_{\Sigma^{r}(u)} \mathcal{H}^{n-r}(\partial u(x)) \mathrm{d} \mathcal{H}^{r}(x) \leq\left(\begin{array}{l}
n \\
r
\end{array}\right) L^{n-r} W_{n-r}(\bar{\Omega}) .
$$

Remark. In the previous theorem, it is also possible to consider compact convex subsets $\Omega^{\prime}$ of $\Omega$.

\section{EXAMPLES}

The principal aim of this final section is to show that Theorem 6.3 is a sharp result. This is achieved by constructing a sufficiently large class of examples. The precise result is stated as a theorem.

Theorem 7.1. Let $\Omega$ be a nonempty open bounded convex subset of $\mathbb{R}^{n}$, assume that $L \geq 0$, and let $\epsilon>0$ be chosen arbitrarily. Then, there exists a convex Lipschitz function $u$ defined on $\Omega$, with Lipschitz constant $L$, and such that

$$
\int_{\Sigma r(u)} \mathcal{H}^{n-r}(\partial u(x)) \mathrm{d} \mathcal{H}^{r}(x) \geq\left(\begin{array}{c}
n \\
r
\end{array}\right) L^{n-r} W_{n-r}(\bar{\Omega})-\epsilon,
$$

for all $r \in\{0, \ldots, n\}$.

Proof. The proof is based on the construction of a convex function which vanishes in the interior of a polytope whose boundary is close to $\partial \Omega$ and increases linearly on rays which are normals to the faces of that polytope. We recall that a polytope is the convex hull of finitely many points, and we refer to Schneider [14], Chapters 1 and 2 , for some basic properties of polytopes.

Fix $\delta>0$. There exists a polytope $P, P \subset \Omega$, such that

$$
W_{j}(P) \geq W_{j}(\bar{\Omega})-\delta
$$

for all $j \in\{0, \ldots, n\}$. This fact is true, since every convex body can be approximated, in the Hausdorff metric, by a sequence of convex polytopes (see [14, Theorem 1.8.13), and the quermassintegrals are continuous with respect to this metric.

Let $\mathcal{F}(P)$ denote the collection of all faces of $P$, and let $\mathcal{F}_{j}(P)$ be the subset of $\mathcal{F}(P)$ consisting of all $j$-dimensional faces, for every $j \in\{0, \ldots, n-1\}$. If $A \in \mathcal{F}_{j}(P)$, we denote by $\operatorname{relint}(A)$ the relative interior of $A$ as a $j$-dimensional convex set. Note that every point of $\partial P$ belongs to the relative interior of exactly one face of $P$. Furthermore, we denote by $\nu(P, A)$ the set of all exterior unit normal vectors of $P$ at points of $\operatorname{relint}(A)$. If $A \in \mathcal{F}_{j}(P)$, then $\nu(P, A)$ is the intersection of the unit sphere in $\mathbb{R}^{n}$ with a convex cone of dimension $(n-j)$.

Define

$$
u(x):=L \operatorname{dist}(x, P), \quad x \in \Omega
$$


The function $u$ is convex and nonnegative in $\Omega$, and it vanishes in $P$. Furthermore, let $x \in \Omega \backslash P$, and let $x^{\prime}$ be the nearest point to $x$ on $\partial P$. Then $x=x^{\prime}+s v$ with $v \in \nu(P, A), x \in \operatorname{relint}(A)$, and $s>0$. In this situation, the value attained by $u$ at $x$ is given by the formula

$$
u(x)=L s .
$$

If $x$ is an interior point of $P$, then

$$
\partial u(x)=\{o\} .
$$

If instead $x \in \operatorname{relint}(A)$, for some $A \in \mathcal{F}(P)$, then the following relation holds:

$$
\partial u(x)=\{t v: t \in[0, L], v \in \nu(P, A)\} .
$$

Indeed, let $v \in \nu(P, A)$ and $t \in[0, L]$; let $H$ denote the support hyperplane to $P$ with exterior unit normal vector $v, H^{+}$the open half-space determined by $H$ not containing $P$, and $H^{-}$the complement of $H^{+}$. If $y \in \Omega \cap H^{+}$, then

$$
u(y) \geq L\langle y-x, v\rangle \geq t\langle y-x, v\rangle .
$$

If $y \in \Omega \cap H^{-}$, then

$$
u(y) \geq 0 \geq t\langle y-x, v\rangle .
$$

Hence $t v \in \partial u(x)$, and thus

$$
\partial u(x) \supset\{t v: t \in[0, L], v \in \nu(P, A)\} .
$$

Now let $v^{\prime} \in \partial u(x)$. Then for all $y \in P$ we have that

$$
\left\langle y-x, v^{\prime}\right\rangle \leq 0,
$$

so that $v^{\prime}=t v$ for some $v \in \nu(P, A)$ and some nonnegative $t$. On the other hand, if $s>0$ is such that $x+s v^{\prime} \in \Omega$, by virtue of (20) and by the definition of $\partial u(x)$, we have

$$
L s t=u\left(x+s v^{\prime}\right) \geq\left\langle x+s v^{\prime}-x, v^{\prime}\right\rangle=s t^{2},
$$

whence $t \leq L$. Thus (22) is completely proved.

Finally, it is easy to see that $u$ is Lipschitz with Lipschitz constant $L$.

For every nonnegative $r$, denote by $B(r)$ the ball centred at the origin with radius $r$. Then, with the help of (21) and (22), it is easy to check that for $\rho \geq 0$ the identity

$$
P_{\rho}\left(u, P \times \mathbb{R}^{n}\right)=P+B(\rho L)
$$

holds true.

A consequence of formula (23) is that, for all $\rho \geq 0$,

$$
\mathcal{H}^{n}\left(P_{\rho}\left(u, P \times \mathbb{R}^{n}\right)\right)=\mathcal{H}^{n}(P+B(\rho L))=\sum_{i=0}^{n}\left(\begin{array}{l}
n \\
i
\end{array}\right) \rho^{i} L^{i} W_{i}(P) .
$$

Hence, from a very special case of Theorem 3.1 we get that

$$
F_{i}(u, P)=L^{i} W_{i}(P), \quad i \in\{0, \ldots, n\} .
$$

The quermassintegrals of $P$ can be expressed as follows (see Schneider [14], §4.2):

$$
W_{0}(P)=\mathcal{H}^{n}(P)
$$


and, for $j \in\{1, \ldots, n\}$,

$$
\left(\begin{array}{c}
n \\
n-j
\end{array}\right) W_{j}(P)=\frac{1}{j} \sum_{A \in \mathcal{F}_{n-j}(P)} \mathcal{H}^{j-1}(\nu(P, A)) \mathcal{H}^{n-j}(A) .
$$

Now let us turn to the singular points of $u$. We start with $\Sigma^{n}(u)$. Obviously, relation (21) implies

$$
\int_{\Sigma^{n}(u) \cap P} \mathcal{H}^{0}(\partial u(x)) \mathrm{d} \mathcal{H}^{n}(x)=\mathcal{H}^{n}(P)=W_{0}(P) .
$$

Next we consider $\Sigma^{i}(u)$ for $i \in\{0, \ldots, n-1\}$. From (21) and (22) it follows that a point $x$ of $P$ belongs to $\Sigma^{i}(u)$ if and only if $x \in \operatorname{relint}(A)$ for some $A \in \mathcal{F}_{j}(P)$ with $j \leq i$. Furthermore, let $A$ be an $i$-dimensional face of $P$ and $x \in \operatorname{relint}(A)$. Then, according to (22),

$$
\mathcal{H}^{n-i}(\partial u(x))=\frac{L^{n-i}}{n-i} \mathcal{H}^{n-1-i}(\nu(P, A))
$$

We conclude that

$$
\int_{\Sigma^{i}(u) \cap P} \mathcal{H}^{n-i}(\partial u(x)) \mathrm{d} \mathcal{H}^{i}(x)=\sum_{A \in \mathcal{F}_{i}(P)} \frac{L^{n-i}}{n-i} \mathcal{H}^{n-1-i}(\nu(P, A)) \mathcal{H}^{i}(A),
$$

for $i \in\{0, \ldots, n-1\}$.

From (24) and (25) we infer the relation

$$
\int_{\Sigma^{i}(u) \cap P} \mathcal{H}^{n-i}(\partial u(x)) \mathrm{d} \mathcal{H}^{i}(x)=\left(\begin{array}{c}
n \\
i
\end{array}\right) L^{n-i} W_{n-i}(P),
$$

for $i \in\{0, \ldots, n\}$. Finally, by (19) we get, for every $i \in\{0, \ldots, n\}$, that

$$
\begin{aligned}
\int_{\Sigma^{i}(u)} \mathcal{H}^{n-i}(\partial u(x)) \mathrm{d} \mathcal{H}^{i}(x) & \geq \int_{\Sigma^{i}(u) \cap P} \mathcal{H}^{n-i}(\partial u(x)) \mathrm{d} \mathcal{H}^{i}(x) \\
& \geq\left(\begin{array}{c}
n \\
i
\end{array}\right) L^{n-i} W_{n-i}(\bar{\Omega})-\delta\left(\begin{array}{c}
n \\
i
\end{array}\right) L^{n-i}
\end{aligned}
$$

Since $\delta>0$ was arbitrarily chosen, this concludes the proof of the theorem.

\section{REFERENCES}

[1] G. Alberti, L. Ambrosio, and P. Cannarsa, On the singularities of convex functions, Manuscr. Math. 76 (1992), 421-435. MR 94c:26017

[2] R. B. Ash, Measure, Integration, and Functional Analysis, Academic Press, New York, 1972. MR 55:8281

[3] V. Bangert, Sets with positive reach, Arch. Math. 38 (1982), 54-57. MR 83k:53058

[4] F. H. Clarke, Optimization and Nonsmooth Analysis, Canadian Mathematical Society, WileyInterscience Publication, New York, 1983. MR 85m:49002

[5] D. L. Cohn, Measure Theory, Birkhäuser Boston, Boston, 1980. MR 81k:28001

[6] A. Colesanti, A Steiner type formula for convex functions, Mathematika 44 (1997), 195-214. MR 98h:52018

[7] A. Colesanti and C. Pucci, Qualitative and quantitative results for sets of singular points of convex bodies, Forum Math. 9 (1997), 103-125. MR 98c:32004]

[8] H. Federer, Curvature measures, Trans. Am. Math. Soc. 93 (1959), 418-491. [MR 22:961]

[9] H. Federer, Geometric Measure Theory, Springer, Berlin, 1969. MR 41:1976

[10] J. H. G. Fu, Tubular neighborhoods in Euclidean spaces, Duke Math. J. 52 (1985), 1025-1046. MR 87f:57019 
[11] D. Hug, Generalized curvature measures and singularities of sets with positive reach, Forum Math. 10 (1998), 699-728. MR 99j:52004

[12] P. Kohlmann, Curvature measures and Steiner formulae in space forms, Geom. Dedicata 40 (1991), 191-211. MR 93d:53070

[13] R. T. Rockafellar, Generalized subgradients in mathematical programming, in: Mathematical Programming - The State of the Art, A. Bachem, M. Grötschel and B. Korte (eds.), Proc. of the 11th International Symp. on Mathematical Programming, Bonn, 1982, Springer, Berlin, 1983, pp. 368-390. MR 85b:90089

[14] R. Schneider, Convex Bodies: The Brunn-Minkowski Theory, Encyclopedia of Mathematics and its Applications 44, Cambridge University Press, Cambridge, 1993. MR 94d:52007

[15] N. S. Trudinger, Isoperimetric inequalities for quermassintegrals, Ann. Inst. H. Poincaré, Analyse non Linéaire 11 (1994), 411-425. MR 95k:52013

[16] M. Zähle, Integral and current representation of Federer's curvature measures, Arch. Math. 46 (1986), 557-567. MR 88a:53072

Universitá Degli Studi di Firenze, Dipartimento di Matematica "U. Dini", Viale MorGAGNi 67/A, 50134 Firenze, Italy

E-mail address: colesant@udini.math.unifi.it

Mathematisches Institut, Albert-Ludwigs-Universität, Eckerstrasse 1， D-79104 Freiburg i. Br., Germany

E-mail address: hug@sun1.mathematik.uni-freiburg.de 